\title{
ON POLYNOMIAL INTERPOLATION TO ANALYTIC FUNCTIONS WITH SINGULARITIES*
}

\author{
BY J. L. WALSH
}

Méray has given $†$ the following illustration to show that polynomials formed from a given function by interpolation do not necessarily converge to that function. Interpolate to the function $f(z)=1 / z$ by means of the polynomials $p_{n}(z)$ of respective degrees $\ddagger n=1,2,3, \cdots$, required to coincide with $f(z)$ in the $(n+1)$ th roots of unity; this condition defines the polynomials $p_{n}(z)$ uniquely. Moreover, we have

$$
p_{n}(z) \equiv z^{n},
$$

because the equation $p_{n}(z)=1 / z$ is satisfied provided $z$ is one of the $(n+1)$ th roots of unity. Even though the sequence $p_{n}(z)$ is defined by interpolation from the function $f(z)=1 / z$, the polynomials $p_{n}(z)$ do not approach the function $f(z)$ for $|z|<1$, as $n$ becomes infinite, but approach the limit zero. It is naturally not surprising that these polynomials should fail to approach $f(z)$ for $|z|<1$, since $f(z)$ has a singularity in that region; this sequence of polynomials fails to approach the limit $f(z)$ even in a neighborhood of the curve $|z|=1$ on which interpolation takes place.

In this connection, it is worth while to recall Runge's result $\S$ that if $f(z)$ is analytic for $|z| \leqq 1$, then the sequence of interpolating polynomials $p_{n}(z)$ of respective degrees $n$ which coincide with $f(z)$ in the $(n+1)$ th roots of unity converges to the limit $f(z)$ for $|z| \leqq 1$.

* Presented to the Society, March 25, 1932.

$\dagger$ Annales de l'École Normale Supérieure, (3), vol. 1 (1884), pp. 165-176. This illustration is also presented by Montel (after Méray), in his Séries de Polynomes, 1910, p. 51.

$\ddagger$ A polynomial of the form $a_{0} z^{n}+a_{1} z^{n-1}+\cdots+a_{n}$ is said to be of degree $n$.

$\S$ Theorie und Praxis der Reihen, 1904, p. 137. This method of Runge's has been more systematically developed by Fejér, Göttinger Nachrichten, 1918, pp. 319-331, and by L. Kalmár, Mathematikai és Physikai Lapok, 1926 , pp. 120-149, but only for interpolation in points on the boundary of a region of a function known to be analytic in that region. 
Méray's illustration is of such simplicity, directness, and beauty, and apparently has stood alone for so long, that it seems worth while to furnish it with some companionship. The purpose of the present note is to provide such companionship by the proof of the following theorem.

THEOREM 1. Let $f(z)$ be an arbitrary function continuous for $|z|=1$. Let the polynomials $p_{n}(z)$ of respective degrees $n,(n=1$, $2, \cdots)$, be defined by the requirement of coinciding with $f(z)$ in the $(n+1)$ th roots of unity. Then the sequence $p_{n}(z)$ approaches the limit

$$
f_{1}(z)=\frac{1}{2 \pi i} \int_{|z|=1} \frac{f(t) d t}{t-z}
$$

for $|z|<1$, uniformly for $|z| \leqq r<1$.

Lagrange's interpolation formula for the polynomial $p_{n}(z)$ of degree $n$ which takes on the values $K_{1}, K_{2}, \cdots, K_{n+1}$ at the $n+1$ distinct points $z_{1}, z_{2}, \cdots, z_{n+1}$ is

$$
p_{n}(z)=\sum_{k=1}^{n+1} \frac{K_{k}}{p^{\prime}\left(z_{k}\right)} \frac{p(z)}{z-z_{k}},
$$

where $p(z)=\left(z-z_{1}\right)\left(z-z_{2}\right) \cdots\left(z-z_{n+1}\right)$; the polynomial $p_{n}(z)$ is uniquely determined by these requirements. Under the circumstances of the theorem, we set $z_{k}=\omega^{k}$, where $\omega=e^{2 \pi i /(n+1)}$, $p(z)=z^{n+1}-1$. It follows that we have

$$
p_{n}(z)=\sum_{k=1}^{n+1} f\left(\omega^{k}\right) \frac{\omega^{k}\left(z^{n+1}-1\right)}{(n+1)\left(z-\omega^{k}\right)} .
$$

With the exception of the term $z^{n+1}$ in the numerator, which approaches zero, equation (3) suggests computation of the integral which appears in (2) by division of the circle $C$ defined by $|z|=1$, at the points $\omega^{k}$. We have

$$
f_{1}(z)=\lim _{n \rightarrow \infty} \frac{1}{2 \pi i} \sum_{k=1}^{n+1} \frac{f\left(\omega^{k}\right)\left(\omega^{k+1}-\omega^{k}\right)}{\omega^{k}-z},
$$

(5) $\lim _{n \rightarrow \infty}\left[f_{1}(z)-p_{n}(z)\right]$

$$
=\lim _{n \rightarrow \infty}\left[\frac{1}{2 \pi i}+\frac{z^{n+1}-1}{(n+1)(\omega-1)}\right] \sum_{k=1}^{n+1} \frac{\omega^{k}(\omega-1) f\left(\omega^{k}\right)}{\omega^{k}-z} .
$$


By (4), the summation on the right-hand side approaches the limit $2 \pi i f_{1}(z)$, which is continuous for $|z|<1$, and the limit is approached uniformly for $|z| \leqq r<1$. ${ }^{*}$ The quantity $(n+1)(\omega-1)$ approaches as its limit $2 \pi i$, for we have

$$
\begin{aligned}
\omega & =\cos \frac{2 \pi}{n+1}+i \sin \frac{2 \pi}{n+1}, \\
\frac{(n+1)(\omega-1)}{2 \pi i} & =\frac{\cos \frac{2 \pi}{n+1}-1}{\frac{2 \pi i}{n+1}}+\frac{\sin \frac{2 \pi}{n+1}}{\frac{2 \pi}{n+1}},
\end{aligned}
$$

which approaches the limit unity. The square bracket in the right-hand member of (5) thus approaches zero for $|z|<1$, uniformly for $|z| \leqq r<1$, and the factor of this bracket is bounded uniformly in $z$ and $n,|z| \leqq r<1$, so the proof of our theorem is complete.

The theorem and proof are obviously valid if the given function $f(z)$ is not supposed continuous on $C:|z|=1$, but merely integrable in the sense of Riemann.

The sequence $p_{n}(z)$ clearly converges uniformly in a region containing the curve $C$ in its interior when and only when the given function $f(z)$ or its analytic extension is analytic on and within $C$. The first part of this result was proved by Féjer (loc. cit.), or compare Walsh. $\dagger$ Reciprocally, if the sequence $p_{n}(z)$ converges uniformly for $|z| \leqq \rho>1$, we shall prove $f(z)$ analytic on and within $C$. The limit of the sequence $p_{n}(z)$ is analytic for $|z|<\rho$. The obvious equation $p_{m(n+1)}\left(\omega^{k}\right)=f\left(\omega^{k}\right),(m=1,2, \cdots)$, where $\omega=e^{2 \pi i /(n+1)}$, implies the convergence of the sequence $p_{n}(z)$ to the function $f(z)$ at each point $\omega^{k}$, hence at a set of points everywhere dense on $C$. The continuity of $f(z)$ for $|z|=1$ and the analytic character of the limit of the sequence $p_{n}(z)$ for $|z|<\rho$ then implies the identity on $C$ of those two functions, hence the fact that $f(z)$ or its analytic extension is analytic for $|z|<\rho$. If the sequence $p_{n}(z)$ converges uniformly for $|z| \leqq 1$, the function $f(z)$ is analytic for $|z|<1$, and continuous for $|z| \leqq 1$.

* Compare Runge, Acta Mathematica, vol. 6 (1885), pp. 229-244; Montel, loc. cit., p. 57; or Osgood, Funktionentheorie, 1928, pp. 579-581.

$\dagger$ Transactions of this Society, vol. 34 (1932), pp. 22-74, \$11. 
The following result is a not uninteresting complement to the main theorem already proved.

THEOREM 2. The function $f_{1}(z)$ which is the limit for $|z|<1$ of the sequence $p_{n}(z)$ of the interpolating polynomials for $f(z)$ is also the limit for $|z|<1$, uniformly for $|z| \leqq r<1$, of the sequence of polynomials $P_{n}(z)$ of respective degrees $n=1,2, \cdots$, of best approximation to $f(z)$ on $C$ in the sense of least squares.

The polynomial $P_{n}(z)$ is that polynomial of degree $n$ for which

$$
\int_{C}\left|f(z)-P_{n}(z)\right|^{2}|d z|
$$

is least. Such a polynomial is known to exist and be unique; it is defined* by

$$
\begin{aligned}
P_{n}(z) & =c_{0}+c_{1} z+\cdots+c_{n} z^{n}, \\
c_{n} & =\frac{1}{2 \pi} \int_{C} f(z) \bar{z}^{n}|d z|=\frac{1}{2 \pi i} \int_{C} f(z) \frac{d z}{z^{n+1}} .
\end{aligned}
$$

We have the relation

$$
f_{1}(z)=\frac{1}{2 \pi i} \int_{C} \frac{f(t) d t}{t-z}=\frac{1}{2 \pi i} \int_{C} f(t) d t\left[\frac{1}{t}+\frac{z}{t^{2}}+\frac{z^{2}}{t^{3}}+\cdots\right] .
$$

The infinite series converges uniformly in $z$ and $t$ for $t$ on $C$, $|z| \leqq r<1$, and hence may be integrated term by term. Thus we have from $(6) f_{1}(z)=c_{0}+c_{1} z+c_{2} z^{2}+\cdots$, a series which is uniformly convergent for $|z| \leqq r<1$; this includes the relation to be proved:

$$
f_{1}(z)=\lim _{n \rightarrow \infty} P_{n}(z), \quad|z|<1 .
$$

The polynomial $P_{n}(z)$ is the sum of the first $n+1$ terms of the Maclaurin development of $f_{1}(z)$.

Several particular cases are worth mentioning. If there exists a function analytic for $|z|<1$, continuous for $|z| \leqq 1$, which coincides with the given function $f(z)$ on $C$, then $f_{1}(z)$ naturally coincides with this function. If there exists a function $F(z)$ analytic for $|z|>1$ (including the point $z=\infty$ ), continuous for $|z| \geqq 1$, which coincides with the given function $f(z)$ on $C$, then each $c_{n}(n>0)$ vanishes, and we have

* See for instance Kowalewski, Determinantentheorie, 1909, §137. 


$$
\lim _{n \rightarrow \infty} p_{n}(z)=\lim _{n \rightarrow \infty} P_{n}(z)=c_{0}=F(\infty),|z|<1 ;
$$

here is included the case $f(z)=1 / z^{k}, k$ a positive integer $(k=1$ is Méray's example), and we have $f_{1}(z) \equiv 0$. More generally, if on $C$ the function $f(z)$ can be expressed as $f(z)=f_{1}(z)+f_{2}(z)$, where $f_{1}(z)$ is analytic for $|z|<1$, continuous for $|z| \leqq 1$, and where $f_{2}(z)$ is analytic for $|z|>1$ including the point at infinity, is continuous for $|z| \geqq 1$, and vanishes at infinity, then we have

$$
\lim _{n \rightarrow \infty} p_{n}(z)=\lim _{n \rightarrow \infty} P_{n}(z)=f_{1}(z),|z|<1,
$$

uniformly for $|z| \leqq r<1$. If this function $f_{1}(z)$ is analytic for $|z|<\rho>1$, then we have

$$
\lim _{n \rightarrow \infty} P_{n}(z)=f_{1}(z),|z|<\rho, \text { uniformly for }|z| \leqq \rho^{\prime}<\rho,
$$

no matter what $f_{2}(z)$ may be, but we have

$$
\lim _{n \rightarrow \infty} p_{n}(z)=f_{1}(z),|z|<\rho \text {, uniformly for }|z| \leqq \rho^{\prime}<\rho,
$$

if and only if $f_{2}(z)$ vanishes identically.

Another illustration of the close connection between interpolation in the $(n+1)$ th roots of unity and approximation on $C$ in the sense of least squares has recently been indicated by the present writer (loc. cit.).

If the function $f(z)$ is analytic for $|z|<T>1$, then for the polynomials $p_{n}(z)$ and $P_{n}(z)$ already defined we have

$$
\lim _{n \rightarrow \infty}\left[p_{n}(z)-P_{n}(z)\right]=0,|z|<T^{2} \text {, uniformly for }|z| \leqq R<T^{2}
$$

even though $f(z)$ has singularities for $T \leqq|z|<T^{2}$.

It is not to be supposed (compare Kalmár, loc. cit.) that interpolation in points arbitrarily chosen on $C$ is always equivalent in the sense illustrated to approximation on $C$ in the sense of least squares, even when the points of interpolation $z_{n}^{(k)}$ for the interpolating polynomial $p_{n}(z)$ of degree $n$ are such that the limit of the maximum distance between successive points $z_{n}^{(k)}$ on $C$ for a given $n$ approaches zero with $1 / n$. We give an example to illustrate this fact, where the $n+1$ points of interpolation for the polynomial $p_{n}(z)$ are the roots of 


$$
\left(\frac{1-\alpha z}{\alpha-z}\right)^{n+1}=1, \alpha>1
$$

These points of interpolation are thus the transforms in the zplane of the $(n+1)$ th roots of unity in the $w$-plane, under the transformation

$$
w=\frac{1-\alpha z}{\alpha-z},
$$

which leaves $C$ invariant and transforms the interior and exterior of $C$ respectively into the interior and exterior of $C$. It is simpler to study the situation in the w-plane. We interpolate to the function $f(z)=1 /(w+T), 0<T<1$, in the points $w$, $w^{n+1}=1$, by rational functions $p_{n}(z)=F_{n}(w)$ of respective degrees (in $z$ or $w) n$ whose poles coincide in the points $w=\alpha$. The reader can easily verify the formula

$$
\begin{aligned}
p_{n}(z) & =F_{n}(w) \\
& =\frac{(T+\alpha)^{n}\left(w^{n+1}-1\right)+\left[(-1)^{n}+T^{n+1}\right](w-\alpha)^{n}}{\left[(-1)^{n}+T^{n+1}\right](w+T)(w-\alpha)^{n}}
\end{aligned}
$$

the expression in terms of $z$ is found from (8). It appears from (9) that one may write

$$
p_{n}(z)=\frac{1}{w+T}+\frac{(T+\alpha)^{n}\left(w^{n+1}-1\right)}{\left[(-1)^{n}+T^{n+1}\right](w+T)(w-\alpha)^{n}},
$$

so that, for $|w|<1$, we have actual convergence when and only when the condition

$$
T+\alpha<|w-\alpha|
$$

is satisfied. Condition (10) is equivalent to the condition that $w$ should lie exterior to a certain circle whose center is $\alpha$ and which cuts $C:|w|=1$. Thus the sequence $p_{n}(z)$ converges in only a part of the unit circle $|z| \leqq 1$, and in that part converges to the original function $1 /(w+T)$.

It would be of interest to extend the main theorem of this note to the study of curves other than the unit circle; compare the references already given to Fejér and Kalmár.

HARVARD UNIVERSITY 